\title{
MAC Enhancements to Support Quality of Services (QoS) in Ad Hoc Wireless Networks: EMACA (Enhancement of Multiple Accesses with Collision Avoidance)
}

\author{
Neeraj Kumar Agrawal \\ Student, M.Tech (IT) \\ School of Information Technology \\ Rajiv Gandhi Proudyogiki \\ Vishwavidyalaya
}

\author{
Prof. Sanjeev Sharma \\ Head of Department \\ School of Information Technology \\ Rajiv Gandhi Proudyogiki \\ Vishwavidyalaya \\ Bhopal (M.P.)
}

\begin{abstract}
Ad hoc wireless network (AWN) is a collection of mobile hosts forming a temporary network on the fly, without using any fixed infrastructure [1], [2], [3], [4], [5], [6]. QoS (Quality of Service) is the idea that transmission rates, error rates, and other characteristics can be measured, improved, and to some extent guaranteed in advance in ad hoc network however in particular concern for the continuous transmission of high bandwidth video and multimedia information this kind of content dependably transmitting is difficult in public networks using ordinary "best effort" protocols [1], [2], [3], [4], [7], [8]. MACA (Multiple Accesses with Collision Avoidance) Protocol is a Contention based Sender initiated Protocol which uses Three way handshaking means that RTS - CTS - Data packet exchange [2]. It used in network congestion avoidance to help in determining the correct sending rate by binary exponential back off (BEB) Algorithm in which if a packet transmitted by a node is lost, the node uses the binary exponential back-off (BEB) algorithm to back off a random interval of time before retrying which is then also inadequate trustworthy because of data sending acknowledgement is not received, hence Enhancement of Multiple Accesses with Collision Avoidance (EMACA) Protocol based on MACA with some modifications over it have been proposed. Instead of three way handshaking in MACA, EMACA protocol propose five way handshaking RTS - CTS - DSSI DSSI_ACK - DATA Sending - ACK. A comparative study was done on QualNet 4.0 Version simulator [9], [10] over MACA \& EMACA. AODV routing protocol was used to evaluate the MACA and EMACA performance [4], [5], [11]. Results show that the EMACA simulation performs well as compared to MACA in sense of Throughput, Total Packet Received and Drop Packet Ratio under varying conditions of no. of nodes and pause time.
\end{abstract}

\section{Keywords}

AWNs, QoS, MAC, MACA, EMACA, AODV, Throughput, Total Packet Received and Drop Packet Ratio.

\section{INTRODUCTION}

Mobile Ad Hoc Networks are wireless networks which do not require any infrastructure support for transferring data packet between two nodes [1], [2], [3], [4], [12]. In these networks nodes also work as a router that is they also route packet for other nodes. Nodes are free to move, independent of each other, topology of such networks keep on changing dynamically which makes routing much difficult. Therefore routing is one of the most concerns areas in these networks. Normal routing protocol which works well in fixed networks does not show same performance in Mobile Ad Hoc Networks. In these networks routing protocols should be more dynamic so that they quickly respond to topological changes [1], [3], [4], [5], [12].

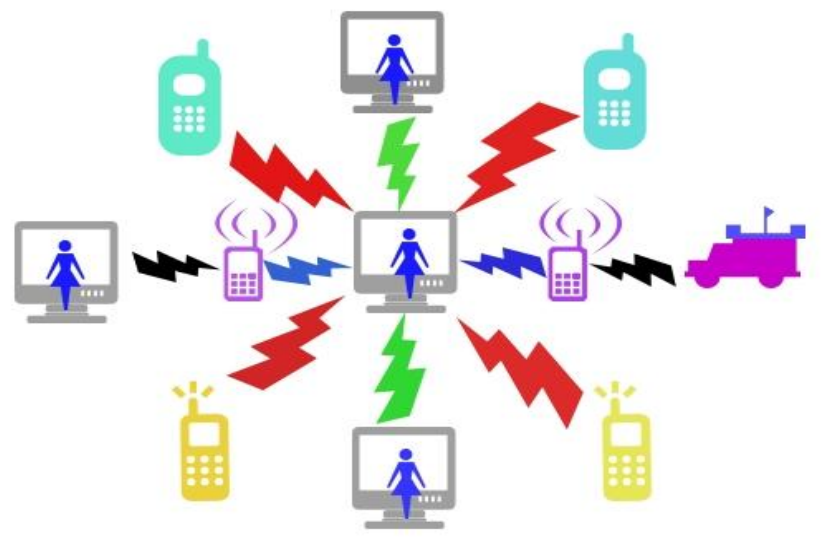

Figure 1: Mobile Ad Hoc Network

Ad hoc wireless networks (AWNs) are zero configurations, self organizing, and highly dynamic networks formed by a set of mobile hosts connected through wireless links [1], [3], [4], [12]. As these are infrastructure less networks, each node should act also as a router. Hence they, the termed "mobile host", "node", and "station" and used interchangeably. As a router, the mobile host represents an intermediate node which forwards traffic on behalf of other nodes. If the destination node is not within the transmission range of the source node, the source node takes help of the intermediate nodes to communicate with the destination node. Tactical communication required on battle-fields, among a fleet of ships, or among a group of armored vehicles are some of the military applications of these networks. Civilian applications include peer-to-peer computing and file sharing, collaborated computing in a conference hall, and search and rescue operations [6].

Quality of service (QoS) is the performance level of a service offered by the network to the user. The goal of QoS provisioning 
is to achieve a more deterministic network behavior, so that information carried by the network can be better delivered and network resources can be better utilized [4], [7], [8]. A network or a service provider can offer different kinds of services to the users. Here, a service can be characterized by a set of measurable Pre specified service requirements such as minimum bandwidth, maximum delay, maximum delay variance (jitter), and maximum packet loss rate. After accepting a service request from the user, the network has to ensure that service requirements of the user's flow are met, as per the agreement, throughout the duration of the flow (a packet stream from the source to the destination) [1], [2], [7], [8].

- Delay: End-to-end delay is the elapsed time for a packet to be passed from a sender through network domains to its intended destination.

- Delay variations (jitter): The variation in end-to-end transient delay is called jitter, also often referred to as delay variation.

- Bandwidth: The maximum data transfer rate that can be sustained between two end points of the network is defined as the bandwidth of the network link.

- Packet Loss rate: is defined as the ratio of dropped packets to the total number of packets.

A MAC protocol in a multi-access medium is essentially a distributed scheduling algorithm that allocates the channel to requesting nodes [2], [4], [12], [13]. Two commonly used access principles in wireless networks are fixed-assignment channel access and random access method. In the former method, a pair of nodes is statically allocated a certain time slot (frequency band or spread spectrum code), as is the case for most of voiceoriented wireless networks. On the other hand, in random access MAC protocols, the sender dynamically competes for a time slot with other nodes. This is a more flexible and efficient method of managing the channel in a fully distributed way, but suffers from collisions and interference.

Medium Access Control (MAC) protocols are responsible for coordinating the access from active nodes [5], [6], [12]. These protocols are of significant importance since the wireless Communication channel is inherently prone to errors and unique problems such as the hidden-terminal problem, the exposedterminal problem, and signal fading effects. Although a lot of research has been conducted on MAC protocols, the various issues involved have mostly been presented in isolation of each other. We therefore make an attempt to present a comprehensive survey of major schemes, integrating various related issues and challenges with a view to providing a big-picture outlook to this vast area. Various MAC protocols developed for wireless ad hoc networks. In contention-free schemes (e.g., TDMA, FDMA, and CDMA) [5], [6]; certain assignments are used to avoid contentions. Contention based schemes, are aware of the risk of collisions of transmitted data. Since contention-free MAC protocols are more applicable to static networks and/or networks with centralized control. In this paper we focus on the contention based MAC protocols. Due to the lack of centralized control in ad hoc networks, researchers have been mainly focused on contention based MAC protocols in this area. [6], [11] Carrier Sense Medium Access (CSMA) one of the earliest mechanisms adopted for ad hoc networks. In CSMA, a transmitter will first sense the wireless channel in the vicinity and refrain itself from transmission if the channel is already in use. Various methods such as ALOHA [6], [11] and n-persistent algorithms can used to determine how long the deferred node should wait before the next attempt. [6], [11] CSMA introduces hidden node and exposed node problems, which are mentioned earlier.

Multiple Access with Collision Avoidance (MACA) is a slotted media access control protocol used in wireless LAN data transmission to avoid collisions caused by the hidden station problem and to simplify exposed station problem [2], [12], [14], [15], [16]. This MACA protocol is not fully solve the hidden node and exposed terminal problem and nothing is done regarding receiver blocked problem.

\section{* Contention Based Protocol}

* Nodes are not guaranteed periodic access to the channel.

* They cannot support real time traffic.

* Three way handshaking.

* RTS - CTS - Data packet exchange

* Binary Exponential back off Algorithm

* Sender initiated Protocol

* RTS-CTS carrier information about the duration of time for neighbor nodes.

The basic idea of MACA is a wireless network node makes an announcement before it sends the data frame to inform other nodes to keep silent. When a node wants to transmit, it sends a signal called Request-To-Send (RTS) with the length of the data frame to send. If the receiver allows the transmission, it replies the sender a signal called Clear-To-Send (CTS) with the length of the frame that is about to receive. Meanwhile, a node that hears RTS should remain silent to avoid conflict with CTS; a node that hears CTS should keep silent until the data transmission is complete.

* When a node wants to transmit a data packet, it first transmits a RTS (Request to Send) frame.

* The receiver node, on receiving the RTS packet, if it is ready to receive the data packet, transmits a CTS (Clear to Send) packet.

* Once the sender receives the CTS packet without any error, it starts transmitting the data packet.

* If a packet transmitted by a node is lost, the node uses the binary exponential back-off (BEB) algorithm to back off a random interval of time before retrying.

Here in section 2 and 3 we have briefly described the Problem Description that is going to be evaluated and proposed algorithm. Consequently In section 4 we have discussed the Experimental Configuration for simulation in the QualNet 4.0 simulator and implementation and results In Section 5. Section 6 explains the Simulation environment used for evaluation of the EMACA protocols. And conclusions have been withdrawn in section 7. In Section 8 we have briefly described the Future Works. 


\section{PROBLEM DESCRIPTION}

The objective research was to evaluate proposed EMACA (Enhancement Multiple Access with Collision Avoidance) Protocol for wireless ad-hoc networks through in given network based on performance. It is a sender initiated protocol. MACA Protocol does not use the new three control messages because there are three problems in this protocol. First one is Hidden terminal and Exposed terminal problems, second one is the congestion problem because more than one source sends the RTS message for transmission and third one is, MACA does not use the Acknowledgement control message, so it's not a reliable. In the pre existing MACA protocol hidden terminal and exposed terminal problems were avoided and not considered crucial [2]. We have to try solving this problem by the implementation of the new protocol EMACA. EMACA Protocol is based on MACA Protocol, so it's only the solution of the pre existing problems. Second control message DSSI (Data Sending for Synchronization Information), its uses for what is Data length and RTS/CTS exchange is a success, Hence defer transmission till data ends. With simple uni-directional transmissions the only relevant congestion is at the receiver; however, with our bidirectional RTS-CTS-DATA message exchange, congestion at both ends of the transmission is relevant. The last control message is ACK, ACK uses for reliability. When TX sends the Data and Rx receive the Data then after completed transmission the Rx sends the ACK for intimation that Data received. At the beginning of this master thesis, implementation of the protocols has been released, so the first main task was to implement some of the protocols.

\section{PROPOSED ALGORITHM}

Let us assume Transceiver (TX) wants to transmit a data packet to Receiver (Rx).

\section{1) The Control rules}

Step1. When TX is in IDLE state and wish to transmit a data packet to $\mathrm{Rx}$, it sets a random timer and goes to the CONTEND state.

Step2. When station $\mathrm{Rx}$ is in IDLE state and receives a RTS packet from TX, it transmits a Clear-To-Send (CTS) packet. Rx then sets a timer and waits for DSSI state.

Step3. When TX is in Wait for CTS state and receives a CTS packet from Rx, it clears the timer, transmits a DSSI (Data Send for Synchronization Information) followed by the data packets to Rx. TX then enters Wait for DSSI-ACK state and sets a timer.

Step4. When Rx is a waiting for DSSI state and receives a DSSI (Data Send for Synchronization Information) packet from TX, transmits a DSSI-ACK packet from Rx. it goes to wait for Data state and sets a timer.

Step5. When TX is in Wait for DSSI-ACK state and receives a DSSI-ACK packet from Rx, transmit data packets from TX, it goes to wait for ACK packet and sets a timer.

Step6. When $\mathrm{Rx}$ is in Wait for Data state and receives a data packet from TX, it clears the timer, transmits an ACK packet, then goes to IDLE state.

Step7. When TX is in Wait for ACK state and receives an ACK packet from $\mathrm{Rx}$, it resets the state to IDLE, and clears the timer. Step8. When Rx is in IDLE state and receives a RTS for a data packet it acknowledged last time, it sends the ACK again instead of CTS.
Step9. If TX receives a RTS packet when it is in CONTEND state, it transmits CTS packet to the sender, goes to the Wait for Data Sending for synchronization Information state and sets a timer value.

Step10. If N2 is in Exit state and receives an RTS, it goes to the Wait for Contend state and sets a timer value.

Step11. If N2 is in Exit state and receives CTS, it goes to the Wait for Contend state and sets a timer value.

Step12. If N2 is in Wait for Contend state and receives an RTS or CTS, the timer value is increased if required.

Step13. If a station waits for RTS state and receives an RTS packet, it transmits a CTS packet to the TX, goes to the wait for DSSI state and sets a timer values.

2) The Deferral rules

Step1. When N2 hears a RTS packet from TX to Rx, it goes from its current state to the Exit state, and sets a timer value sufficient for TX to hear Rx's CTS.

Step2. When N2 hears a Data Sending packet from TX to Rx, it goes from its current state to the Exit state, and sets a timer sufficient for TX to transmit the Data packet and then hear Rx's ACK.

Step3. When N1 hears a CTS packet from Rx to TX, it goes from its current state to the Exit state, and sets a timer value sufficient for Rx to hear TX's data.

\section{3) The Timeout rules}

Stepl. When a station is in Wait for Contend state and the timer expires, it sets a random timer and goes to the CONTEND state. Step2. When a station is in CONTEND state and the timer expires, it may either transmit a RTS packet to perform a senderinitiated data transmission (TX).

For sender-initiated transmission, A transmits a RTS packet, containing the station ID's of TX and Rx, and the requested number of bytes to send. TX goes to wait for CTS state, and sets a timer value.

Step3. When a timer expires, a station goes to the IDLE state and resets the timer value.

\section{EXPERIMENT CONFIGURATION}

In this paper, we use QualNet 4.0 [9], [10] to simulate our proposed protocol and evaluate the performance of EMACA with MACA.

\subsection{Parameter}

We simulated a scenario of 50 mobile nodes active in a square area of $1500 \mathrm{~m} \times 1500 \mathrm{~m}$. Nodes move inside with random waypoint mobility, the maximum velocity is $10 \mathrm{~m} / \mathrm{s}$. In this model a mobile node is initially placed in a random location in the simulation area, and then moved in a randomly chosen direction between $[0,2]$ at a random speed between $\left[\right.$ Speed $_{\text {Min }}$, Speed $_{\text {Max }}$. The movement proceeds for a specific amount of time or distance, and the process is repeated a predetermined number of times. In this work in variable pause time have been chosen Min speed $=0 \mathrm{~m} / \mathrm{s}$, Max speed $=10 \mathrm{~m} / \mathrm{s}$. All the simulation work was carried out using AODV routing protocol. Network traffic is provided by using Constant Bit Rate (CBR) sources. A CBR traffic source provides a constant stream of packets throughout the whole simulation, thus further stressing the routing task. 
CBR (Constant Bytes Rate) stream has been selected between the nodes. The size of each packet is 512 bytes and the sending rate of source is 1 packet per second. As soon as the mobile node reaches its destination, it will stop for a short period of time, which is 10 second, 20s, 30s, 40s, 50s, 60s, 80s and 100s, and then selects a destination randomly to move until the simulation ends. Simulation time has been set to 100 seconds.

\begin{tabular}{|c|c|c|}
\hline S.No. & Parameters & Values \\
\hline 1 & Area & $1500 \mathrm{mx} 1500 \mathrm{~m}$ \\
\hline 2 & Number of nodes & $10,20,30,40$ and 50 Nodes \\
\hline 3 & Application & CBR (Constant Bit Rate) 2 to 3 Nodes \\
\hline 4 & Mobility Model & Random Waypoint \\
\hline 5 & Pause Time & $\begin{array}{c}10,20,30,40,50,60,80 \text { and } 100 \\
\text { Seconds }\end{array}$ \\
\hline 6 & Data Packet & $\begin{array}{c}\text { Constant, } 512 \text { bytes packet size, } 1 \\
\text { packet/s }\end{array}$ \\
\hline 7 & Simulation Time & Constant, 100 Seconds \\
\hline 8 & Max. Speed & Constant, 10 m/s \\
\hline 9 & MAC Protocols & CSMA, MACA and EMACA Protocols \\
\hline 10 & Routing Protocols & AODV \\
\hline 11 & Node Placement & Random \\
\hline 12 & Seed & 1 \\
\hline
\end{tabular}

Table 1: Parameters Value

\subsection{Performance Metrics}

1) Throughput (bits/s):- Throughput is the measure of the number of packets successfully transmitted to their final destination per unit time. It is the ratio between the numbers of sent packets vs. received packets [4], [10], [17].

2) Total Packets received: - Packet delivery ratio is calculated by dividing the number of packets received by the destination through the number of packets originated by the application layer of the source (i.e. CBR source). It specifies the packet loss rate, which limits the maximum throughput of the network. The better the delivery ratio, the more complete and correct is the routing protocol [4], [10], [17].

3) Drop Packet Ratio:- Packet drop ratio is calculated by subtract to the number of data packets sent to source and number of data packets received destination through the number of packets originated by the application layer of the source (i.e. CBR source) [4], [10], [17].

\section{Implimentation AND results \\ 5.1 Effect of Varying Number of Nodes}

Number of nodes may be another varying parameter as it plays important role in performance. Figure 2 (2a, 2b, 2c) shows various performance parameters versus number of nodes. From figure we can observe that routing overload for all protocol increased as number of nodes increased but among them AODV performed poorer as this might be due to flooding of routing packets. While in case of less number of nodes all protocols performed poorer in terms of delivery ratio as nodes breakage may be more and no route may be available.

\subsection{Effect of Varying Pause Time}

Pause time can be defined as time for which nodes waits on a destination before moving to other destination. We used this as a parameter as it is measure of mobility of nodes. Low pause time means node will wait for less time thus giving rise to high mobility scenario. Figure 3 (3a, 3b, 3c) shows various performance parameters versus pause time when other parameters were constant. From figure we can observe that normalized overload for AODV is almost constant. This is because of their reactive nature due to which they offer constant routing overhead in all cases. While for reactive protocols considered here as we increased pause time routing overload has decreased .This is because as routing pause time increases mobility decreases and thus link breakage become rare which in turn will decrease number of route request from sources and hence decreasing overhead. In case of failure in one route other route will be used rather than initiating route request. Also from figure we can see that average delay for EMACA protocol was better at high mobility as they use route already in the table, and no time is required to find route as opposite to MACA protocols as they will wait for route formation. But at lower mobility, we can observe that reactive protocols performed better in terms of average delay among which AODV outperformed MACA. This is because MACA may not use optimum path always unlike AODV. While Throughput (bits/s) for MACA and EMACA was near to $100 \%$ with MACA performing better because of multiple path information in its route cache (AODV always stores best path). For evaluation of MACA and EMACA in simulation, AODV routing protocol was used [4], [5], [11].

Figure 2: Various Performance Parameter V/s Numbers of Nodes

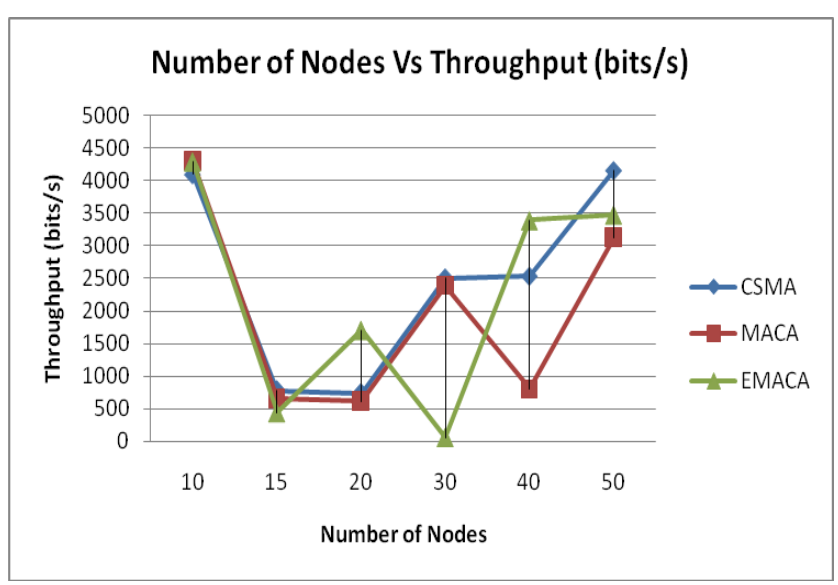

Figure 2 (a): Number of Nodes Vs Throughput (bits/s) 


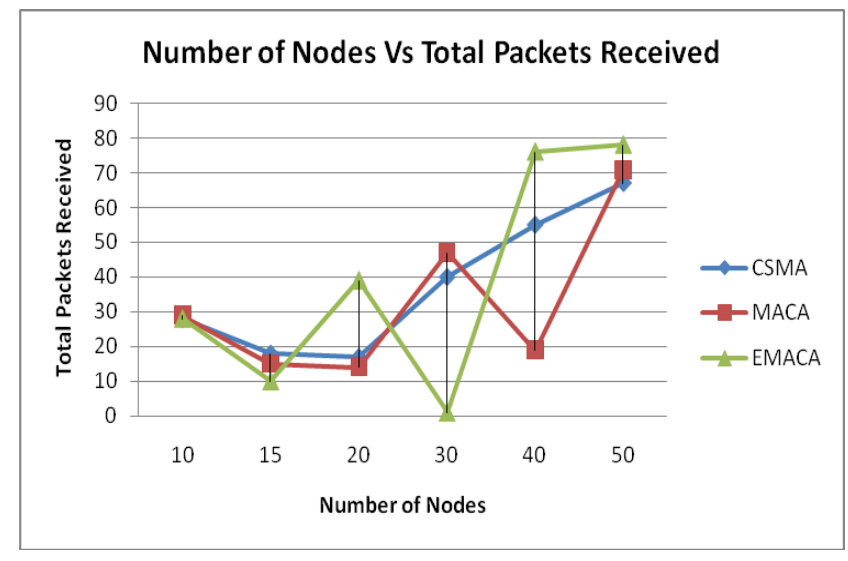

Figure 2 (b): Number of Nodes Vs Total Packets Received

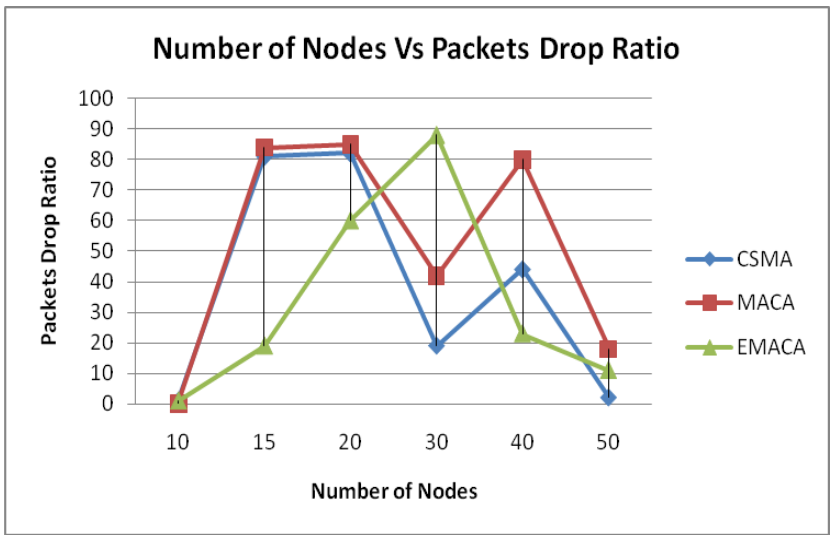

Figure 2 (c): Number of Nodes Vs Packets Drop Ratio

Figure 3: Various Performance parameters versus Pause Time

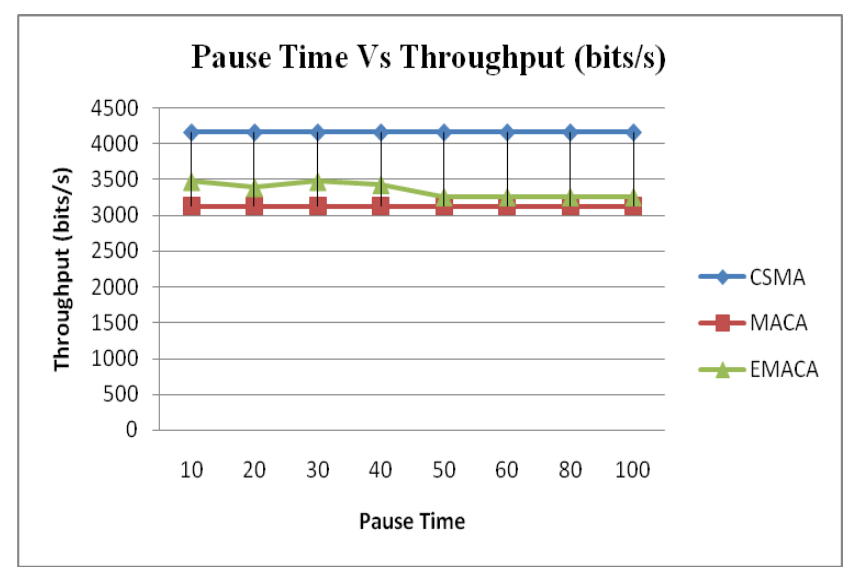

Figure 3 (a): Pause Time Vs Throughput (bits/s)

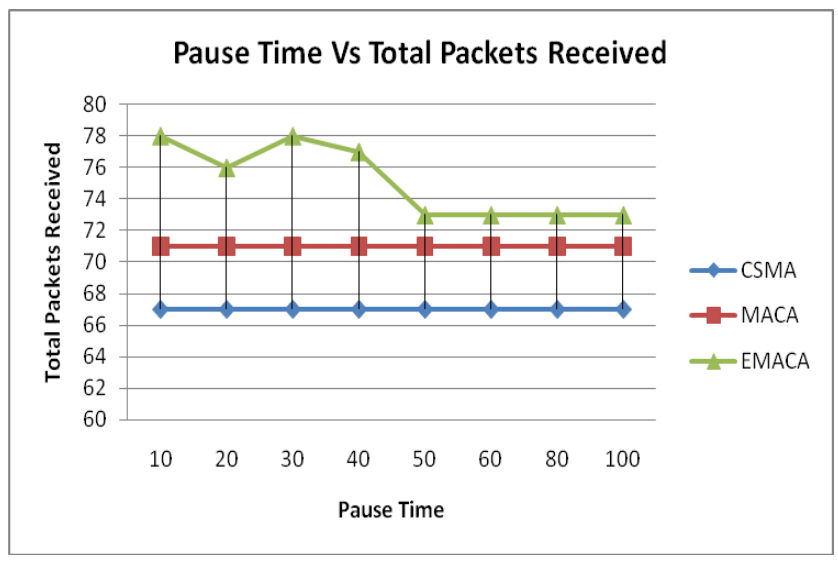

Figure 3 (b): Pause Time Vs Total Packets Received

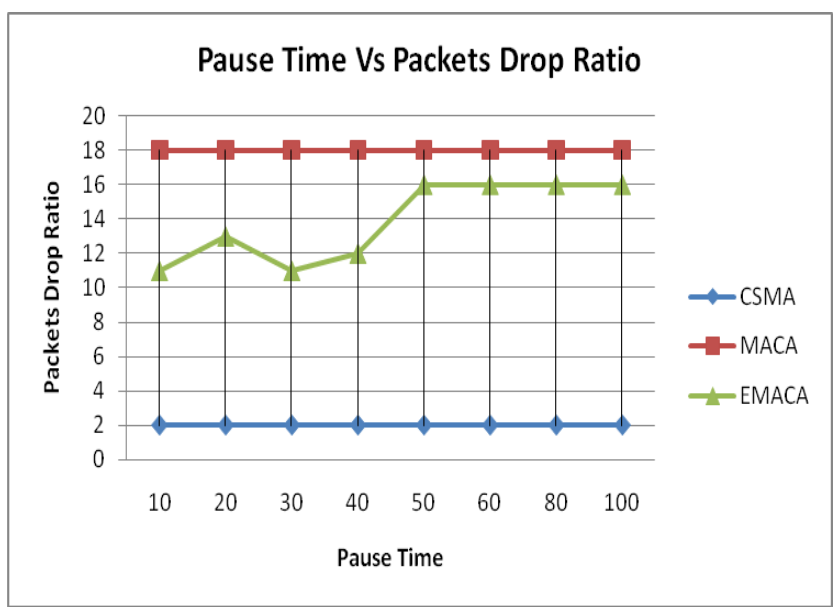

Figure 3 (c): Pause Time Vs Packets Drop Ratio

\section{Evaluation of emaca protocol}

In this scenario, the number of nodes has varied from 10 to 50 nodes with constant pause time and node speeds. Throughput is the measure of the number of packets successfully transmitted to their destination per unit time. The ratio between the number of sent packets and received packets has increased for different node density because the source node and destination node were connected to the circuit switching, which is more reliable. Analysis and comparison of throughput for CSMA, MACA and EMACA Protocols with varying node density and pause time was represented in figure 2(a) and 3(a) respectively. EMACA performs well as compare to the MACA protocol. So EMACA is reliable as compare to the MACA, because the ratio between the number of send packets and the number of received packets has increased, so in this case the reliability of the protocol has increased. But In other case the CSMA and MACA results have been consistent but EMACA has not been consistent results. But throughput has increased as compare to the MACA so EMACA protocol is reliable, but it is not $100 \%$ reliable because EMACA is not consistent. So EMACA is not fully reliable but compare to the MACA, it is reliable. The Analysis of Total Packets 
Received for CSMA, MACA and EMACA Protocols under varying node density and pause time was represent in figure 2(b) and 3(b) respectively And The total packets received means that the CBR source sends the data packets for CBR destination. In this case the total packets received ratio has increased as compare to another protocol. So the communication of CBR source and CBR destination is more secure due to reason of any other intermediate node can not be interrupted, which is not in route. Analysis of Packets Drop Ratio for CSMA, MACA and EMACA protocols with varying node density and pause time were represented in figure 2(c) and 3(c) respectively. Then the case of EMACA the drop packets ratio has decrease because EMACA uses more overhead as compare to MACA. From results it can be observed that EMACA is reliable but not consistent while CSMA and MACA are consistent but not reliable because of drop packet ratio in EMACA protocol is less than MACA.

\section{CONCLUSIONS}

The Efficient MAC protocols can provide significant benefits to mobile ad hoc networks, in terms of both performance and reliability. The issues associated with the design of a MAC protocol for wireless ad hoc networks are: node mobility; an error- prone, broadcast and shared channel; time-synchronization; bandwidth efficiency; QoS support. Many MAC protocols for such networks have been proposed so far but their performance in terms of Throughput, Total packet received and drop packet ratio is questionable and is not satisfactory. Hence EMACA was proposed, which performance is superior in terms of Throughput, Total packets received and Drop packet ratio. To enhance the performance of MACA, EMACA has been introduced which using DSSI, DSSI-ACK and ACK as the backup route when the original route is no longer in use and solved the problem of data packets which are unnecessary to resend. The intention of the mechanism is to reduce the waiting time of data transmission before route is reestablished. In doing so, the Drop Packet Ratio will be reduced. Besides, the Throughput (bits/s) will be enhanced. And the Total Packet Received will be enhanced as compare to the MACA protocol. The simulation results show that the new protocol EMACA has found to be better performance than MACA protocol. Preliminary simulation results presented here validate the operational correctness of EMACA and show the potential for significant throughput improvement (at least in selected topologies). So EMACA protocol is more reliable. Consequently it identified and rectified additional performance drawbacks in EMACA through the use of adaptive learning strategies and better physical layer capabilities.

\section{FUTURE WORK}

Although EMACA performs well under different conditions but how much of the savings from avoided collisions in EMACA will spent on RTS/CTS and DSSI/DSSI-ACK overhead given typical modem turnaround times and data packet sizes? How much better does power-controlled EMACA perform than the basic EMACA scheme? Simulations gave further confidence in the protocol and showed further possible improvements in performance. The protocol can also be improved by adding carrier sensing. Simulations suggest that this could improve utilization under heavy load by about $5 \%$. From the results it is expected that RRTS, a new control message addition will abolish the expose terminal and hidden terminal problems and EMACA in multi casting application may be potential future outcome.

\section{ACKNOWLEDGMENTS}

I am deeply indebted to my supervisor Prof. Sanjeev Sharma, HOD School of Information Technology whose help, stimulating suggestions and encouragement helped me in all the time of research.

\section{REFERENCES}

[1] T. Bheemarjuna Reddy, I. Karthigeyan, B.S. Manoj, C. Siva Ram Murthy, "Quality of service provisioning in ad hoc wireless networks: a survey of issues and solutions", Department of Computer Science and Engineering, Indian Institute of Technology, Madras 600036, India, Ad Hoc Networks 4 (2006) 83-124.

[2] Imrich Chlamtac, Marco Conti, Jennifer J.N. Liu, "Mobile ad hoc networking: imperatives and challenges", University of Texas at Dallas, Dallas, TX, USA, Ad Hoc Networks 1 (2003) 13-64.

[3] C.K. Toh, "Ad Hoc Mobile Wireless Networks: Protocols and Systems", Springer Prentice Hall Publishers, ISBN 013 $0078174,2001$.

[4] Azzedine Boukerche, "Algorithms and Protocols for Wireless and Mobile Ad Hoc Networks", University of Ottawa, Ottawa, Canada, ISBN 978-0-470-38358-2 (cloth) TK5105.77.A44 2008.

[5] Arun Kumar B. R., "Performance Comparison of Wireless Mobile Ad-Hoc Network Routing Protocols", Bangalore \& Research Scholar, Dept. of CS, School of Science \& Technology, Dravidian University, Kuppam-517425, A. P., India, June 2008.

[6] S Krishnamurthy, "Ad-Hoc Networks - Technologies and Protocols - P Mohapatra", Springer Publishers, 2004 (ISBN: 0-387-22689-3).

[7] Changzhou Wang, Guijun Wang, Haiqin Wang, Alice Chen, "Quality of Service (QoS) Contract Specification, Establishment, and Monitoring for Service Level Management", Rodolfo Santiago Boeing Phantom Works Seattle, WA, USA 0-7695-2743-4/06, 2006.

[8] El-Bahlul Fgee, Jason D. Kenney, William J. Phillips, William Robertson, "Comparison of QoS performance between IPv6 QoS management model and IntServ and DiffServ QoS models", Dalhousie University, Department of Engineering Mathematics, and Halifax, ISBN:0-76952333-1, 2005.

[9] QualNet 4.5 Programmer's Guide, Scalable Network Technologies, Inc., 6701 Center Drive West, Suite 520, Los Angeles, CA 90045. 
[10] Scalable Network Technologies, "QualNet simulator 4.0Version",tutorial on http://www.cs.binghamton.edu / vinkolar/QualNet/qualnet-tut1.pdf.

[11] C.E. Perkins, “Ad Hoc Network", Pearson Education, ISBN: 8131720969, 2008.

[12] C. Siva Ram Murthy and B. S. Manoj, "Ad Hoc Wireless Networks: Architectures and Protocols", Pearson Education, ISBN13: 9780131470231 ISBN10: 0-13- 147023-X, 2008.

[13] Blerta Bishaj, "MAC protocols, contention-based with reservation and scheduling", Helsinki University of Technology.

[14] Bartlomiej Zielilski, "Contention MAC Protocols Efficiency Testing in a Small Wireless Network", Silesian Technical University, Bartlomiej.
[15] Alan Demers, Scott Shenker, Lixia Zhang, "Media Access Protocol for Wireless LAN's", University of California at Berkeley.

[16] Akhilesh Kumar Dubey Anjana Jain S.V. Charhate, "Performance Evaluation of MAC layer Protocols for Ad hoc WLAN", 23 Park Road Indore (M.P.) INDIA DOI 10.1109/ICETET.2008.66.

[17] Jun-Zhao Sun Media Team, "Mobile Ad Hoc Networking: An Essential Technology for Pervasive Computing", University of Oulu, Finland. 